\title{
庆大霉素特异性单链DNA适配体的篮选、表征和 应用
}

\author{
巫朦朦 ${ }^{1}$, 韩旭艳 ${ }^{1}$, 蔡蓉风 ${ }^{1}$, 李茂林 ${ }^{2 *}$, 田亚平 ${ }^{1}$, 周楠迪 ${ }^{1 *}$ \\ 1. 江南大学生物工程学院, 糖化学与生物技术教育部重点实验室, 无锡 214122 \\ 2. 宜兴市肿瘤医院检验科, 宜兴 214200 \\ *联系人, E-mail: 13961511114@163.com; zhounandi@jiangnan.edu.cn \\ 收稿日期：2018-10-31; 接受日期：2018-12-25; 网络版发表日期：2019-02-18 \\ 国家自然科学基金(批准号: 31271860)、江苏省六大人才高峰(批准号: JY-078)和国家轻工技术与工程一流学科自主课题(批准号: LITE2018- \\ 07)资助
}

\begin{abstract}
摘要庆大霉素是一种常用的氨基糖苷类抗生素, 对细菌尤其是革兰氏阴性菌有显著的抑菌作用, 但其本身具有 较强的毒副作用，而不规范的使用会对环境和人体健康产生危害。因此，建立一种高灵敏的快速检测庆大霉素的 方法对于加强食品市场监管和环境监控具有重要意义. 本文利用基于Sepharose亲和层析的指数富集的配体系统 进化技术，经过九轮篎选和克隆测序，从体外合成的全长为79个核苷酸的随机单链DNA文库中篎选得到34条与 庆大霉素有亲和力的适配体序列. 通过同源性分析将这些序列分为 6 个家族, 利用Mfold软件分析这些序列的二级 结构及其稳定性, 从中选出结构稳定性最高的序列进行解离常数 $\left(K_{\mathrm{d}}\right)$ 测定, 最后得到一条与庆大霉素具有极高亲 和力的适配体Ap-26, 其解离常数 $\left(K_{\mathrm{d}}\right)$ 为 $14.00 \pm 3.34 \mathrm{nmol} / \mathrm{L}$. 通过Autodock 4.0软件对 Ap-26与庆大霉素进行分子对 接模拟, 结果表明Ap-26与庆大霉素主要的结合位点是17 20, 54 56位核苷酸. 在此基础上, 利用适配体Ap-26为识 别元件构建了基于金纳米粒子(gold nanoparticles, AuNPs)比色检测庆大霉素的方法, 验证了该适配体的应用潜力. 当庆大霉素的浓度在50 700 nmol/L范围内时, AuNPs溶液在520 nm处的吸光值与庆大霉素的浓度存在良好的线 性关系，检测限为 $11.50 \mathrm{nmol} / \mathrm{L}$. 将该方法应用于牛奶样品中庆大霉素的检测时发现牛奶基质成分对检测结果的 影响很小, 庆大霉素的回收率均超过 $90 \%$. 以上结果表明本文節选得到的庆大霉素适配体能有效应用于庆大霉素 检测方法的构建.
\end{abstract}

关键词庆大霉素, 指数富集的配体系统进化技术, 适配体, 亲和层析, 金纳米粒子

庆大霉素(gentamicin)是小单狍菌属(Micromonospora sp.)发酵产生的一组结构相近的多组分氨基糖苷 类抗生素 ${ }^{[1]}$. 庆大霉素主要用于治疗细菌感染, 尤其是 革兰氏阴性菌引起的感染 ${ }^{[2]}$ ，其作用机制是通过与细
菌核糖体30S小亚基上的 $16 \mathrm{~S}$ rRNA结合，引起遗传密 码的误读, 阻断细菌蛋白质的合成, 并破坏细胞膜的完 整性, 从而抑制细菌活性 ${ }^{[3]}$. 庆大霉素在我国曾因抗菌 谱较广、抑菌效果好、价格低廉且易得的优点而被广

引用格式: 巫朦朦, 韩旭艳, 蔡蓉风, 等. 庆大霉素特异性单链DNA适配体的篮选、表征和应用. 中国科学: 生命科学, 2019, 49: 637-648 Wu M M, Han X Y, Cai R F, et al. Selection, characterization and application of gentamicin-specific single-stranded DNA Aptamers (in Chinese). Sci Sin Vitae, 2019, 49: 637-648, doi: 10.1360/N052018-00238 
泛应用于临床, 后因其严重的毒副作用, 特别是耳毒性 和肾毒性以及导致肠道菌群失调引发相关肠道类疾病 而逐步被其他类型抗生素所取代 ${ }^{[4]}$. 但目前仍广泛用 于动物呼吸道和消化道疾病的治疗, 对奶牛子宫内膜 炎和乳房炎也有很好的治疗效果 ${ }^{[5]}$. 由于庆大需素的 不规范使用会带来动物源性食品和环境中庆大霉素残 留过量的问题，目前我国对庆大霉素的残留进行了严 格的限定，规定内脏、牛奶和奶粉中的庆大霉素最大 残留量为 $20 \mu \mathrm{g} / \mathrm{kg}$, 肉类和水产品为 $10 \mu \mathrm{g} / \mathrm{kg}$. 因此, 建 立简便、快速、高灵敏度的庆大霉素检测方法对于食 品安全监管和环境监测等有着重要意义. 目前, 与其他 抗生素的检测类似，常规的庆大需素检测方法主要有 微生物检测法、免疫检测法、仪器分析法等 ${ }^{[8 \sim 10]}$, 这 些方法存在操作复杂、成本高、耗时长、灵敏度低等 缺点, 难以达到快速、准确地检测庆大霉素的要求, 亟 需建立新型有效和经济的庆大霉素检测方法.

近年来，以适配体(aptamer)为识别元件的生物传 感检测法因特异性高、灵敏度高，能有效克服常规检 测方法的缺点而备受关注. 适配体又称为适配子，是 通过指数富集的配体系统进化技术(systematic evolution of ligands by exponential enrichment, SELEX)从 随机葟核苷酸文库中篮选得到的一类能够与靶分子高 选择性、高特异性结合的单链DNA(ssDNA)或RNA 自1990年Tuerk等人 ${ }^{[11]}$ 和Ellington等人 ${ }^{[12]}$ 首次提出适 配体这一概念及篮选方法以来，越来越多的适配体序 列得到报道. 适配体在空间上具有一定的延展性，当 与靶标相互作用时，其在静电引力、氢键、范德华力 和碱基堆积等作用下能够自身折叠形成复杂的结构, 如G-四聚体、凸环、发夹、茎和环等，从而形成与靶 物质形状匹配、紧密契合的结构 ${ }^{[13]}$. 作为一种新型的 分子识别元件，适配体与抗体相比有其独特的优 势 ${ }^{[14 ~ 17]}$ : (i ) 靶标范围更广, 包括离子、抗生素和毒 素等小分子物质; (ii) 篮选周期短, 在较短时间内就能 获得符合预期的适配体序列; (iii) 可在体外快速大量 地合成且批次之间的差异较小，制备过程更加简单; （iv）易于修饰，可与其他分子或功能基团连接; ( $\mathrm{v}$ ) 适配体分子量小、体积小，组织渗透性更好；(vi）与 目标物具有与抗体相当或更高的亲和力和特异性. 目 前靶向抗生素的适配体已经陆续有报道，包括特异性

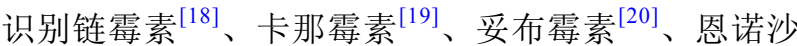

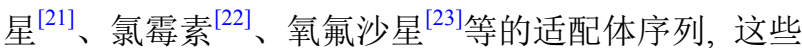

适配体也已被用于构建多种类型的抗生素检测方法， 包括电化学法 ${ }^{[24,25]}$ 、比色法 ${ }^{[26]}$ 、荧光法 ${ }^{[27]}$ 、化学发 光法 ${ }^{[28]}$ 等. 与微生物检测和理化检测等常规方法相比, 基于适配体的检测方法具有更高的灵敏度; 而与基于 抗体的免疫分析法相比，适配体的特点赋予其检测方 法构建的多样性和灵活性.

纳米粒子是指尺寸在纳米级别的超微粒子，其具 备一些特有性质，如量子尺寸效应、表面效应和非线 性光学效应等，因此表现出与宏观物截然不同的磁

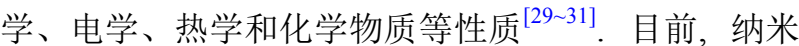
粒子由于其极强的吸附力和较好的生物相容性而被广 泛应用于适配体生物传感器的构建. 其中, 金纳米粒子 (AuNPs) 是迄今研究和使用最多的一种金属纳米粒

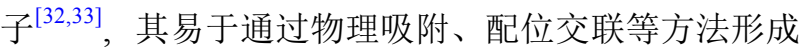
金标记物，在医学诊断、成像、生物传感器、催化和 药物运输等方面得到了广泛的应用 ${ }^{[34-36]}$. AuNPs溶液 也称胶体金溶液，是一种带负电的胶体溶液，在静电 斥力作用下具有良好的稳定性。但在高盐溶液中, AuNPs的稳定性会遭到破坏而发生聚集，该过程中溶 液也会由酒红色逐渐加深至蓝色或灰色，从而被广泛 用于建立基于AuNPs的比色分析方法. 该方法通过灵 敏的颜色变化来检测靶分子，具有可视化、简便快 捷、灵敏度高等优点.

迄今为止，尚无庆大霉素特异性ssDNA适配体的 报道，本文利用基于Sepharose亲和层析的SELEX法从 初始随机文库中篮选出与庆大霉素有高亲和力的 ssDNA适配体，进一步对适配体的结构和结合特性进 行表征，并利用适配体为分子识别元件构建了快速检 测庆大霉素的AuNPs比色法.

\section{1 材料与方法}

\section{1 材料}

随机ssDNA初始文库为: 5'-TAGGGAATTCGTCGACGGATCC-N35-CTGCA GGTCGACGCATGCGCCG-3'，两端为固定序列，用于PCR扩增时 与引物配对,中间为 35 个碱基的随机序列; PCR扩增引 物两对, 序列如下:

引物I-1: 5'-TAGGGAATTCGTCGACGGAT-3';

引物I-2: 5'-FAM-TAGGGAATTCGTCGACGGAT-3'; 
引物II-1: 5'-CGGCGCATGCGTCGACCTG-3';

引物II-2: 5'-biotin-CGGCGCATGCGTCGACCTG$3^{\prime}$. 以上序列均由生工生物工程(上海)股份有限公司合 成. $N$-差基琥珀酰亚胺 $(N$-hydroxysuccinimide, NHS)、 活化的琼脂糖凝胶(NHS-activated Sepharose 4FF) 购自 GE公司(美国). 链霉亲和素磁珠(Dynabeads M-280)购 自Dynal公司(美国).

\section{2 亲和层析柱的准备}

亲和层析介质的制备方法主要参考Song 课题 组 ${ }^{[37]}$ 的实验方法, 具体流程如下: 将 $15 \mathrm{~mL}$ NHS活化的 琼脂糖凝胶和 $10 \mathrm{~mL}$ 溶于偶联缓冲液 $(0.2 \mathrm{~mol} / \mathrm{L} \mathrm{NaH}-$ $\left.\mathrm{CO}_{3}, 0.5 \mathrm{~mol} / \mathrm{L} \mathrm{NaCl}, \mathrm{pH} 8.0\right)$ 的 $10 \mathrm{mmol} / \mathrm{L}$ 庆大霉素混 匀, $28^{\circ} \mathrm{C}$ 轻微摇晃 $4 \mathrm{~h}$. 然后, 将偶联后的琼脂糖凝胶用 偶联缓冲液清洗 5 遍, 加入封闭缓冲液 $(0.1 \mathrm{~mol} / \mathrm{L}$ Tris$\mathrm{HCl}, \mathrm{pH} 8.5), 37^{\circ} \mathrm{C}$ 轻微摇晃封闭 $6 \mathrm{~h}$. 封闭完成后, 用 3 倍柱体积的去离子水清洗琼脂糖凝胶, 再依次通过清 洗液 $1(0.5 \mathrm{~mol} / \mathrm{L} \mathrm{NaCl}, 0.1 \mathrm{~mol} / \mathrm{L}$ 乙酸-乙酸钠, $\mathrm{pH}$ $4.0)$ 、去离子水、清洗液 $2(0.1 \mathrm{~mol} / \mathrm{L}$ Tris- $\mathrm{HCl}$, $0.5 \mathrm{~mol} / \mathrm{L} \mathrm{NaCl}, \mathrm{pH} 4.0)$ 和去离子水重复清洗两次. 最 后将偶联了庆大霉素的琼脂糖凝胶重新悬浮于 $5 \mathrm{~mL}$ 结合缓冲液 $(20 \mathrm{mmol} / \mathrm{L}$ Tris- $\mathrm{HCl}, 50 \mathrm{mmol} / \mathrm{L} \mathrm{NaCl}$, $5 \mathrm{mmol} / \mathrm{L} \mathrm{MgCl}_{2}, 5 \mathrm{mmol} / \mathrm{L} \mathrm{KCl}, 1 \mathrm{mmol} / \mathrm{L} \mathrm{CaCl}_{2}, \mathrm{pH}$ 7.6), 装柱备用.

\section{3 体外篮选}

筛选流程主要包括 4 个步骤, 即捊育、分离、洗 脱、扩增. 具体过程如下. (i ) 孵育: 将 $1.5 \mathrm{nmol}$ ssDNA文库溶于 $5 \mathrm{~mL}$ 结合缓冲液中, $95^{\circ} \mathrm{C}$ 变性 $10 \mathrm{~min}$, 迅速冰浴 $5 \mathrm{~min}$ ，室温放置 $10 \mathrm{~min}$; 再将其加入到经结 合缓冲液预平衡过的亲和层析柱中, 室温孵育 $1 \mathrm{~h}$. (ii) 分离: 孵育结束后, 利用结合缓冲液进行洗 涤, 除去未与庆大霉素结合的ssDNA. (iii) 洗脱: 通过 洗脱缓冲液 $(20 \mathrm{mmol} / \mathrm{L}$ Tris- $\mathrm{HCl}, 50 \mathrm{mmol} / \mathrm{L} \mathrm{NaCl}, 5$ $\mathrm{mmol} / \mathrm{L} \mathrm{MgCl}_{2}, 5 \mathrm{mmol} / \mathrm{L} \mathrm{KCl}, 1 \mathrm{mmol} / \mathrm{L} \mathrm{CaCl}_{2}, 10$ $\mathrm{mmol} / \mathrm{L}$ 庆大霉素, $\mathrm{pH}$ 7.6)将结合在凝胶上的ssDNA洗 脱下来，收集洗脱液获得与庆大霉素特异性结合的 ssDNA; (iv) 扩增: 将上述特异性结合的ssDNA作为 模板以引物 I -1和引物 II - 2 进行PCR扩增，并用 $3 \%$ 的 琼脂糖凝胶电泳验证扩增结果.

\section{4 ssDNA次级文库的制备}

取 $25 \mu \mathrm{L}$ 链霉亲和素修饰过的磁珠, 用结合与清洗 (binding\&washing, B\&W)缓冲液 $(10 \mathrm{mmol} / \mathrm{L}$ Tris- $\mathrm{HCl}$, $1 \mathrm{mmol} / \mathrm{L}$ EDTA, $2 \mathrm{~mol} / \mathrm{L} \mathrm{NaCl}, \mathrm{pH}$ 7.5)清洗3遍, 再加 入 $20 \mu \mathrm{L}$ PCR产物(双链中一条含生物素修饰)和 $80 \mu \mathrm{L}$ $\mathrm{B} \& \mathrm{~W}$ 缓冲液, 轻微振荡, 室温反应 $1 \mathrm{~h}$. 然后, 将其置于 磁性分离架上，弃上清液。用 $\mathrm{B} \& \mathrm{~W}$ 缓冲液清洗 3 遍后, 加入 $30 \mu \mathrm{L}$ 的 $0.1 \mathrm{~mol} / \mathrm{L} \mathrm{NaOH}$, 在 $37^{\circ} \mathrm{C}$ 水浴锅中变性 $2 \mathrm{~h}$, 洗脱从磁珠上释放的单链, 重复两次, 收集洗脱液 即可用作次级文库进行下一轮篮选.

\section{5 次级文库结合率的测定}

篮选得到的ssDNA次级文库用引物 I - 2 和 II - 2 进 行PCR 扩增, 扩增产物通过链霉亲和素磁珠法获得 FAM标记的ssDNA次级文库. 取 4 pmol FAM标记的 ssDNA次级文库, 加入 $100 \mu \mathrm{L}$ 结合缓冲液, 混合均匀于 $95^{\circ} \mathrm{C}$ 变性 $10 \mathrm{~min}$, 迅速冰浴 $5 \mathrm{~min}$, 室温放置 $10 \mathrm{~min}$. 实 验组加入 $10 \mu \mathrm{L}$ 的 $10 \mu \mathrm{mol} / \mathrm{L}$ 庆大霉素溶液, 对照组加 入等体积的结合缓冲液, 并用结合缓冲液分别将体系 补充至 $250 \mu \mathrm{L}$. 常温轻微摇晃 $1 \mathrm{~h}$ 后, 向实验组和对照 组分别加入 $5 \mu \mathrm{L}$ 氧化石墨烯溶液, 混匀后轻微摇晃 $2 \mathrm{~h}$. 将混合液于 $4{ }^{\circ} \mathrm{C}, 8000 \mathrm{r} / \mathrm{min}$ 离心 $10 \mathrm{~min}$, 取上清液 $200 \mu \mathrm{L}$, 利用多功能酶标仪测定苂光值(激发波长 $494 \mathrm{~nm}$, 发射波长 $520 \mathrm{~nm}$ ). 实验组与对照组荧光值的 差值即为 ssDNA次级文库与庆大霉素结合的荧光 强度。

\section{6 篮选产物的克隆、测序和序列分析}

经9轮篮选得到的 ssDNA文库用引物 I - 1 和引 物 II -1进行PCR扩增, 产物胶回收纯化后连接到pMD19T载体, 转化JM109感受态细胞, 挑取阳性转化子送 生工生物工程(上海)股份有限公司进行测序.

对测序成功的适配体序列进行分析, 用DNAMAN 软件分析其一级结构的同源性, 并用Mfold软件对其二 级结构进行模拟, 最优适配体使用Autodock 4.0软件与 庆大霉素分子进行对接模拟，预测出适配体与庆大霉 素作用的主要核苷酸位点.

\section{7 适配体解离常数 $\left(K_{\mathrm{d}}\right)$ 的测定和特异性验证}

取不同浓度梯度的FAM标记的适配体溶液( 0 
$500 \mathrm{nmol} / \mathrm{L})$ 与固定浓度的庆大霉素 $(400 \mathrm{nmol} / \mathrm{L})$ 室温 孵育 $1 \mathrm{~h}$ 后, 加入氧化石墨烯(graphene oxide, GO)室温 孵育 $2 \mathrm{~h}$. GO 能特异性地吸附游离的ssDNA, 而不能吸 附与庆大霉素结合的适配体DNA. 将上述混合物于 $4^{\circ} \mathrm{C}, 8000 \mathrm{r} / \mathrm{min}$ 离心 $10 \mathrm{~min}$, 取上清液. 然后, 用 $200 \mu \mathrm{L}$ 结合缓冲液重悬沉淀, 再以相同的条件取上清液. 最后 将两次离心所得上清液置于 96 孔板中，用多功能酶标 仪测其荧光强度. 根据测量得到的苂光值, 利用GraphPad Prism5.0软件进行非线性回归分析，根据公式: $Y=B_{\max } \times X /\left(K_{\mathrm{d}}+X\right)$ 计算出 $K_{\mathrm{d}}$ 值 $\left(B_{\max }\right.$ 为最大结合位点的 数目, $X$ 为适配体浓度, $Y$ 为荧光强度).

为了验证篎选获得的适配体与庆大霉素结合的特 异性，分别用卡那霉素、新霉素、四环素、氨苄青霉 素、妥布霉素与过量的适配体进行孵育. 依次用多功 能酶标仪测定其结合的ssDNA的苂光强度.

\section{8 金纳米粒子比色法检测庆大霉素}

采用柠檬酸三钠还原法制备AuNPs ${ }^{[20]}$. 将 $100 \mu \mathrm{L}$ 的AuNPs溶液与 $100 \mu \mathrm{L}$ 的 $100 \mathrm{nmol} / \mathrm{L}$ 庆大霉素适配体 混匀，室温避光反应 $1 \mathrm{~h}$, 然后向上述混合物中加入 $50 \mu \mathrm{L}$ 不同浓度的庆大霉素标准溶液 $(0 \sim 700 \mathrm{nmol} / \mathrm{L})$, 避光反应 $50 \mathrm{~min}$. 最后向反应物中加入 $50 \mu \mathrm{L}$ 的 $200 \mathrm{mmol} / \mathrm{L} \mathrm{NaCl}$ 溶液, 并进行紫外-可见吸收光谱表
征，利用 $520 \mathrm{~nm}$ 处的吸光度与庆大霉素浓度之间的关 系对庆大霉素进行检测.

为了证明该方法的实用性，将其应用于人工污染 的牛奶样品中庆大霉素的检测. 首先向市售牛奶中添 加不同浓度的庆大霉素标准溶液 (50，100，200， $500 \mathrm{nmol} / \mathrm{L}$ ), 再滴加 $20 \%$ 三氯乙酸调节 $\mathrm{pH}$ 至 4.6 , 然后 $45^{\circ} \mathrm{C}$ 水浴 $10 \mathrm{~min}$ 沉淀牛奶样品中的蛋白, 再以 $10000 \mathrm{r} / \mathrm{min}$ 离心 $20 \mathrm{~min}$ 除去凝结的蛋白质和脂肪，即 得到经预处理的含庆大霉素的牛奶样品. 将该样品作 为检测液, 按上述方法进行检测.

\section{2 结果}

\subsection{SELEX篮选}

庆大霉素适配体篎选的流程如图1所示. 当ssDNA 文库加样到层析柱中与庆大霉素修饰的琼脂糖凝胶孵 育后，没有与庆大霉素结合的大部分ssDNA随结合缓 冲液流出，只有与庆大霉素有亲和力的序列结合在柱 子上. 将结合序列洗脱后, 以不带修饰的上游引物和 生物素修饰的下游引物进行PCR扩增, 并通过生物素链霉亲和素的相互作用, 将扩增产物dsDNA结合到磁 珠上，加 $\mathrm{NaOH}$ 变性后释放出的ssDNA即可作为下一 轮篮选的文库.

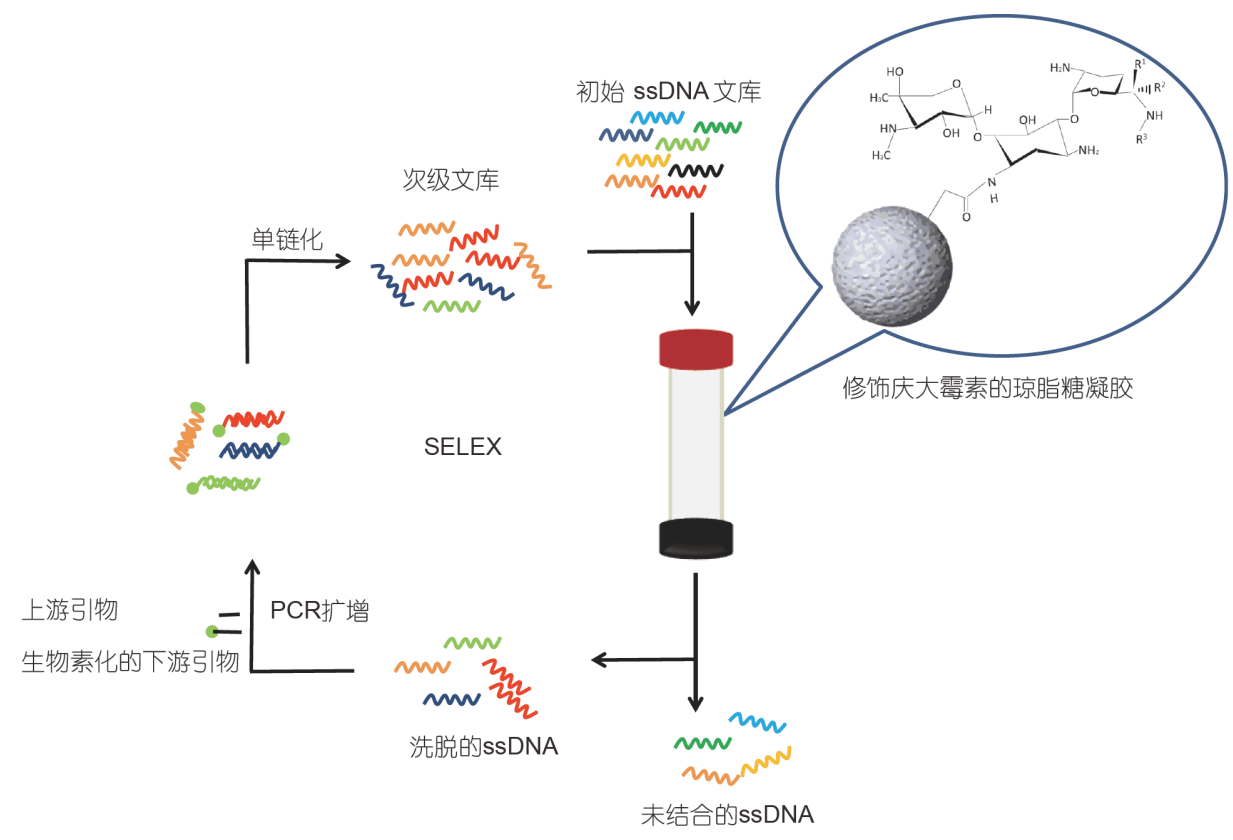

图 1 基于亲和层析的庆大霉素适配体笁选原理图

Figure 1 A schematic procedure of the SELEX process using affinity chromatography 
在SELEX篮选过程中，每一轮的篎选产物通过苂 光法测定ssDNA文库与庆大霉素的结合率. 结合率越 高，表明所得ssDNA 与庆大霉素的亲和力越高，篮选 一直进行到结合率达到饱和为止. 如图2A所示, 随着 篮选轮数的增加, ssDNA文库与庆大霉素的结合率越 来越高, 当篎选到第九轮时, 结合率达到最大值且趋 于平稳，表明此时庆大霉素适配体的富集已经达到饱 和. 因此, 将第九轮篎选所得的ssDNA文库克隆并测 序. 图2B是第一轮和第九轮篮选得到的PCR产物的聚 丙烯酰胺凝胶电泳图，与第一轮相比，第九轮篮选结 束后PCR产物的电泳条带更加集中且明亮.

\section{2 测序结果分析}

将第九轮篎选得到的适配体进行克隆、测序，得 到34条序列. 根据同源性和二级结构的相似性，将这 34 条序列分为 6 个家族，如表 1 所示. 从 34 条序列中选 择序列较多的家族中结构最稳定 $(\Delta G$ 值最低)的序列, 序列 $5 ， 8 ， 22$ 和 26 作为庆大霉素最优适配体的候选序 列, 进行FAM标记适配体的合成和解离常数的测定.

\section{3 适配体的亲和力测定和特异性分析}

采用荧光分析法测定候选适配体序列与庆大霉素 的亲和力. 根据测定的荧光强度, 拟合饱和曲线并得出 解离常数. 如图 $3 \mathrm{~A}$ 所示, 序列 $5,8,22$ 和 26 的 $K_{\mathrm{d}}$ 值分别为
$74.50 \pm 9.77, \quad 80.25 \pm 9.04,96.34 \pm 18.96$ 和 $14.00 \pm$ $3.34 \mathrm{nmol} / \mathrm{L} . K_{\mathrm{d}}$ 值的大小代表序列与靶标亲和力的高 低, $K_{\mathrm{d}}$ 值越小, 表明该序列与靶标的亲和力越高. 因此, 序列26被选作最佳的庆大霉素适配体, 命名为Ap-26.

根据亲和力的考察结果, 对Ap-26的特异性进行验 证. 将Ap-26分别与庆大霉素、卡那霉素、新霉素、四 环素、氨苄青霉素和妥布霉素孵育, 用氧化石墨烯吸 附未与抗生素结合的ssDNA序列, 离心取上清液进行 苂光强度检测. Ap-26与不同抗生素结合的苂光强度 如图3B所示，与庆大霉素结合的适配体荧光强度显著 高于其他抗生素. 因此, Ap-26是庆大霉素的高亲和力 和高特异性适配体序列。

\section{4 最佳适配体的结构分析}

用Autodock 4.0软件对Ap-26与庆大霉素进行分子 对接模拟, 确定出Ap-26与庆大霉素作用时的主要核苷 酸位点. 如图4A所示, 庆大霉素与Ap-26作用的主要位 点是17 20和54 56 号核苷酸. 利用Mfold在线软件分 析Ap-26的二级结构, 如图4B所示, 形成了3组茎环状 结构, 红色矩形框内的核苷酸为预测的庆大霉素结合 位点，其中17 20号核苷酸位于其中一个茎环结构的 双螺旋区内, 而54 56号核苷酸位于单链区内, 推测这 两个区域在进一步形成空间结构时构成了庆大霉素的 结合位点.
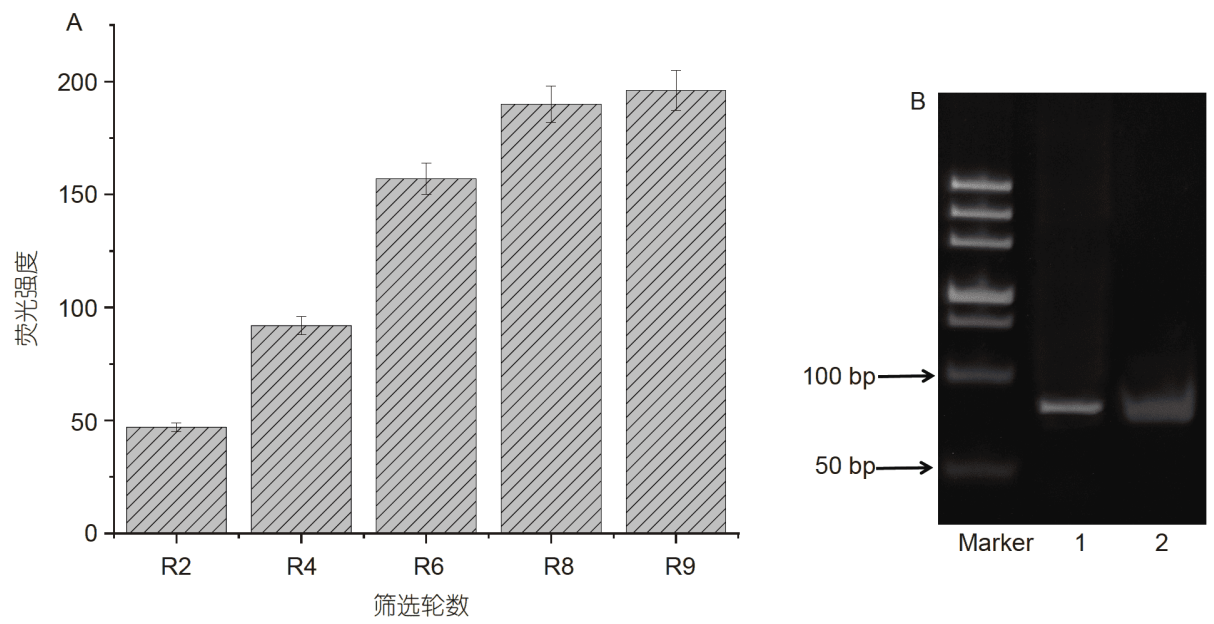

图 2 庆大霉素适配体節选轮数的确定. A: SELEX篮选过程中结合庆大霉素的ssDNA的苂光强度; B: 第一轮和第九轮篮选后 PCR产物的聚丙烯酰胺凝胶电泳图(1: 第九轮笁选产物; 2: 第一轮筛选产物)

Figure 2 Determination of the selection rounds of gentamicin aptamer. A: Fluorescent intensity of gentamicin bound ssDNA after each SELEX round. B: Polyacrylamide gel electrophoresis image for PCR products after the first round and the ninth round of selection (1: The ninth round; 2: the first round) 
表 1 庆大霉素适配体的随机序列和家族分类

Table 1 Random sequence and family classification of gentamicin aptamers

\begin{tabular}{|c|c|c|c|}
\hline 家族 & 中间 35 个随机序列 & 编号 & $\Delta G(\mathrm{kcal} / \mathrm{mol})$ \\
\hline \multirow{5}{*}{ I } & AATGAATAGCGGGCTGTACTGTGGGTCAGCCACCC & 1 & -9.34 \\
\hline & CGCATAAGTGGCGTTCGCAGCGAGATAGGAACCTC & 2 & -9.40 \\
\hline & CATCACCGGCAAGGGTAGCCCTCGATTTCCATCGT & 3,4 & -9.71 \\
\hline & GTTCTGTACGCGAACCGGTCCTTGGATCGTAATCG & 5 & -11.98 \\
\hline & GTAATTCGGACCGGAACTATTCGCGTTTGCATTAT & 6 & -11.23 \\
\hline \multirow{11}{*}{ II } & GCACCGTGACTTTTG GTTCTCTTTCGCGCCAAGTC & 7 & -6.83 \\
\hline & CGGGAACGGGGGCTAAGAACTTGGGAACTGCGCCT & 8,9 & -11.66 \\
\hline & GCGTCCGTAATACATTTCTGTTCGGTTGTCCGACT & 10 & -9.34 \\
\hline & GGCTCTGTAAAATTATATCCGATCGTATTACCCTC & 11 & -6.76 \\
\hline & CCCTTCTTAAATTGTCTAGGTTCCCTTCTTTCTAT & 12 & -6.46 \\
\hline & GCAAGTATATTGTGTTGGTCCTTCTCTGAGAGGTA & 13 & -7.74 \\
\hline & CGCCTATACCTAAGCGCCTTGATTCGACTTTCATC & 14 & -11.08 \\
\hline & ACCCTGGCGTAAAGGGTTTTGCATAAGCTTACACA & 15 & -8.55 \\
\hline & CGCCATATTCCATTCTAGCTTCTAATTTGCGGACG & 16 & -7.88 \\
\hline & ACATTCGTTGAGGATTCTGGCTTCT TCCTTGTACC & 17 & -7.38 \\
\hline & GACTGGTACTTCCTTTGGTGTGTACGAATTGCGCG & 18 & -9.90 \\
\hline \multirow{5}{*}{ III } & TGTTGGTGTGAGCGTGCCGTAATATGCGAGATTTT & 19 & -6.92 \\
\hline & TTTGCCAATCCCATGGTGTTCTTCTAGTCCAGGTT & 20 & -5.65 \\
\hline & GTTCTATATCGATTGTCTCGGCATCAGTTCTCATC & 21 & -7.21 \\
\hline & TGTATATGCAACGTCTCCGCTCTGGTACTTTGTAT & 22 & -10.5 \\
\hline & CTGTTAGCCTAGTTTTCAGTCGCAGTTGCTTCCCT & 23 & -9.35 \\
\hline \multirow{5}{*}{ IV } & AGGCTTTCAAACAGGTCCTACCGCTGAGTTTGACA & 24 & -8.45 \\
\hline & ACATTGGCATGTAAGTCGTATCGCTACTCGCGGGT & 25 & -7.66 \\
\hline & CATCGTCTTCTTGAAGTGTGTTCTCAATAGCGTGG & 26 & -9.08 \\
\hline & CGCATCCTTCTCTAGCGAGGTCTTGCCCAATCATG & 27 & -7.62 \\
\hline & TTAGCCGGTTAGTATTTGCTTGATGGTGGGCACGT & 28 & -6.61 \\
\hline \multirow{4}{*}{$\mathrm{V}$} & AGGTCTCCGAATCCGCTAGTACTAATTTCAGGTTC & 29 & -7.01 \\
\hline & AGTTGTCTAATGGTGATTGTACTAGTAAAGGGACA & 30 & -6.81 \\
\hline & GTCCAATCCTACTCAAGACGATACAGAGTACAATG & 31 & -7.08 \\
\hline & CTTGCCATACGATAAGCAGTTGTTTACGTGTCTCT & 32 & -9.54 \\
\hline \multirow{2}{*}{ VI } & CCGGCGGGGGGTTTTAATCCTTCCTGTGTATTAGG & 33 & -10.03 \\
\hline & GGTTGATGTCGACCCCTATTTGACCTTCGTCTTGT & 34 & -9.60 \\
\hline
\end{tabular}

\subsection{AuNPs比色法检测庆大霉素}

为了考察笁选得到的 $A p-26$ 在庆大霉素残留检测 中的应用性能, 构建了基于适配体Ap-26的AuNPs比色 法检测庆大霉素. 研究表明, ssDNA可以在静电引力等 的作用下，包裹在AuNPs的表面，使得AuNPs在高浓度
盐离子(如钠离子、钾离子等)的溶液中保持稳定而不 会发生聚集反应 ${ }^{[37]}$. 在此条件下, AuNPs溶液由于表面 等离子共振作用而呈现为酒红色，且在 $520 \mathrm{~nm}$ 处有最 大吸收. 而裸露的AuNPs在较高浓度的盐离子存在下 会发生聚集，溶液颜色由原来的酒红色变为紫色或蓝 色甚至是蓝灰色. 当Ap-26与AuNPs㐿育后, 其均匀覆 

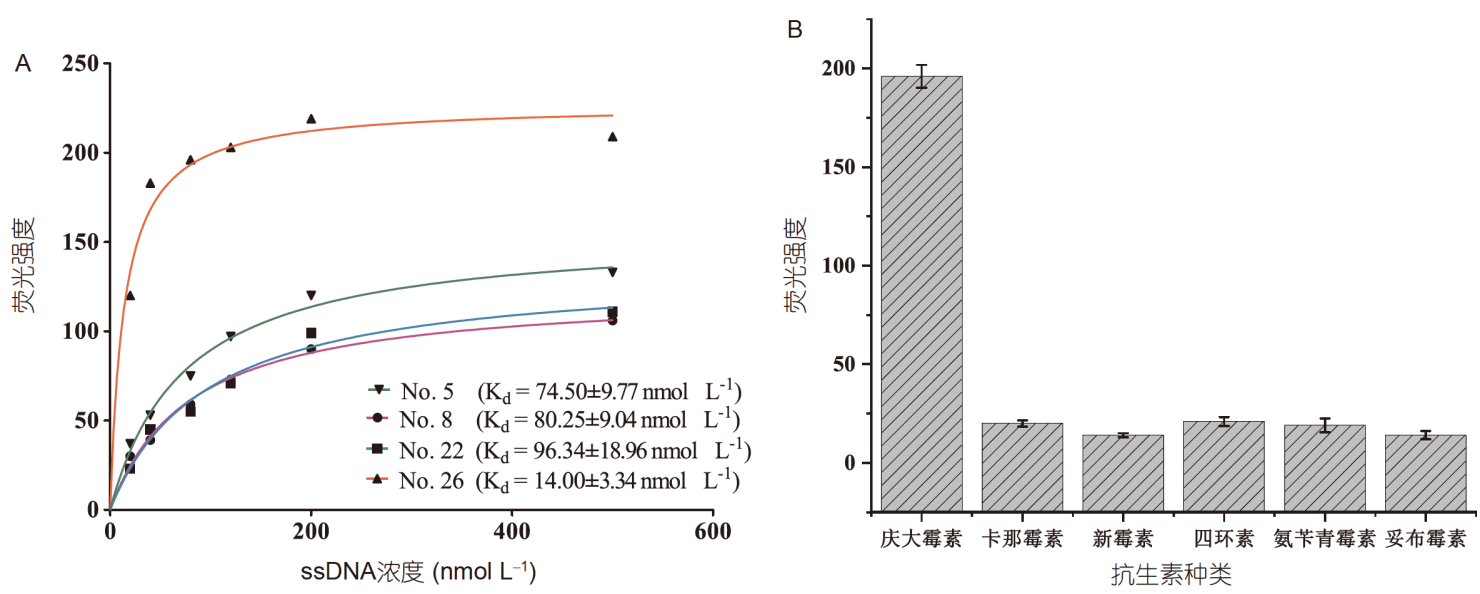

图 3 庆大霉素适配体的亲和力和特异性分析. A: ssDNA与庆大霉素的饱和曲线; B: Ap-26的特异性表征

Figure 3 Analysis of the affinity and specificity of gentamicin aptamers. A: The saturation curves for the dissociation constants of ssDNA towards gentamicin. B: Characterization of specificity of Ap-26
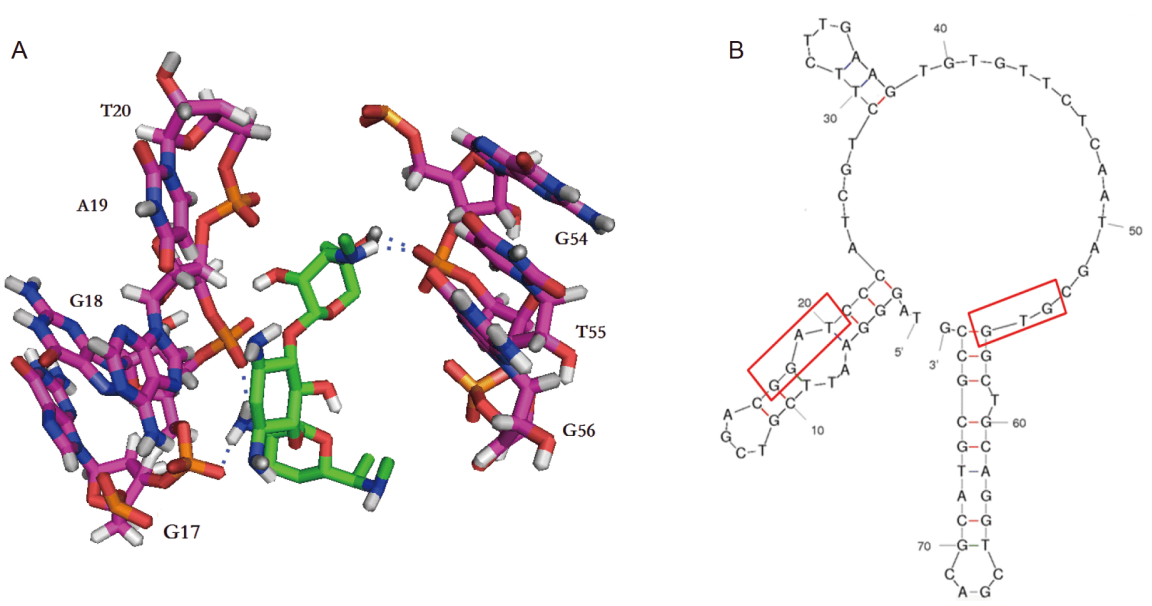

图 4 适配体Ap-26的结构分析. A: 庆大霉素与Ap-26分子对接模拟图; B: Ap-26的二级结构预测

Figure 4 Structural analysis of Ap-26. A: Molecular docking of AP-26 with gentamicin. B: Secondary structure of Ap-26

盖在AuNPs表面，使AuNPs在一定的盐浓度下仍然保 持分散状态而颜色不会发生变化．但当庆大霉素存在 时，其能够竞争性地与AuNPs表面的Ap-26结合，使得 适配体从AuNPs表面脱离，AuNPs表面形成裸露状态， 在高盐溶液中会发生聚集，颜色也会发生变化。并且 随着庆大霉素浓度的升高，其可以特异性地与更多 AuNPs表面的适配体结合，从而使AuNPs表面暴露程 度更高，在高浓度 $\mathrm{NaCl}$ 作用下，溶液颜色逐步由原来 的酒红色变为紫色、蓝色甚至蓝灰色，如图5A所示, 因而通过肉眼观察，即可实现庆大霉素的定性和半定 量测定. 为了实现庆大霉素的定量检测，对AuNPs溶 液的UV-Vis光谱进行了分析，如图5B所示，AuNPs在
$520 \mathrm{~nm}$ 处的吸光度值也随着庆大霉素浓度的不断增加 而逐步下降. 进一步考察了AuNPs溶液在 $520 \mathrm{~nm}$ 处的 吸光度与庆大霉素浓度的关系，如图5C所示，当庆大 霉素的浓度在50 700 nmol/L范围内时, AuNPs溶液在 $520 \mathrm{~nm}$ 处的吸光值随着庆大霉素浓度的增大而逐渐减 小, 呈现良好的线性关系, 线性方程为: $y=-2.849 \mathrm{e}-4 x$ $+0.8528 \quad\left(R^{2}=0.9897\right)$, 该方法的检测限达到 $11.50 \mathrm{nmol} / \mathrm{L}$, 低于国家标准中最低残留量要求. 为了 验证AuNPs比色法检测庆大霉素的特异性, 依次向Ap26 修饰的AuNPs中加入 $600 \mathrm{nmol} / \mathrm{L}$ 的庆大霉素、卡那 霉素、新霉素、四环素、氨苄青霉素以及妥布霉素溶 液, 再加入高浓度的 $\mathrm{NaCl}$ 溶液, 如图5D所示, 加入庆大 
A
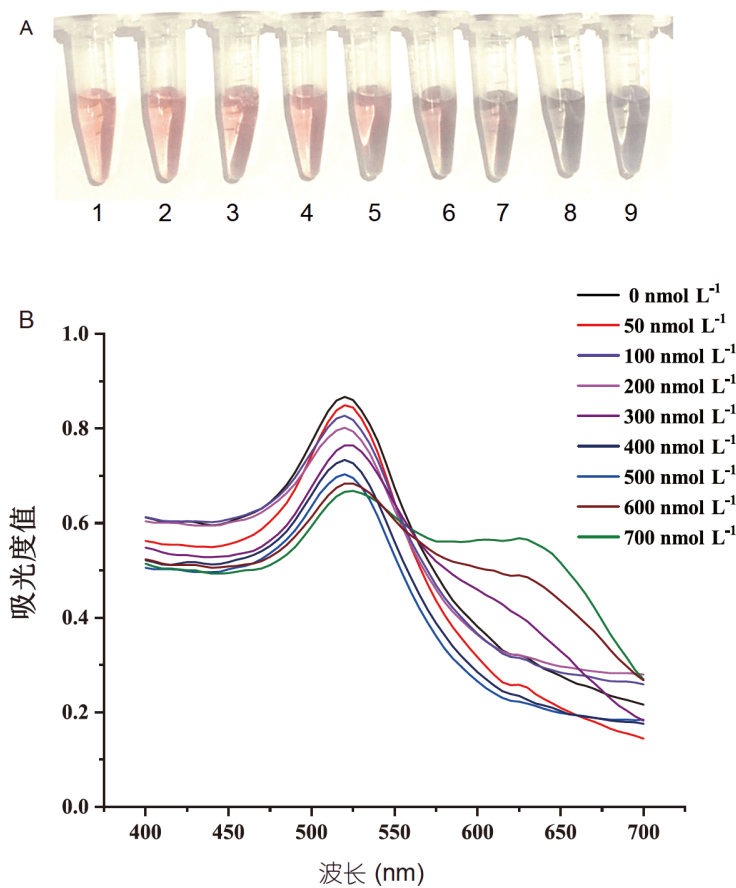
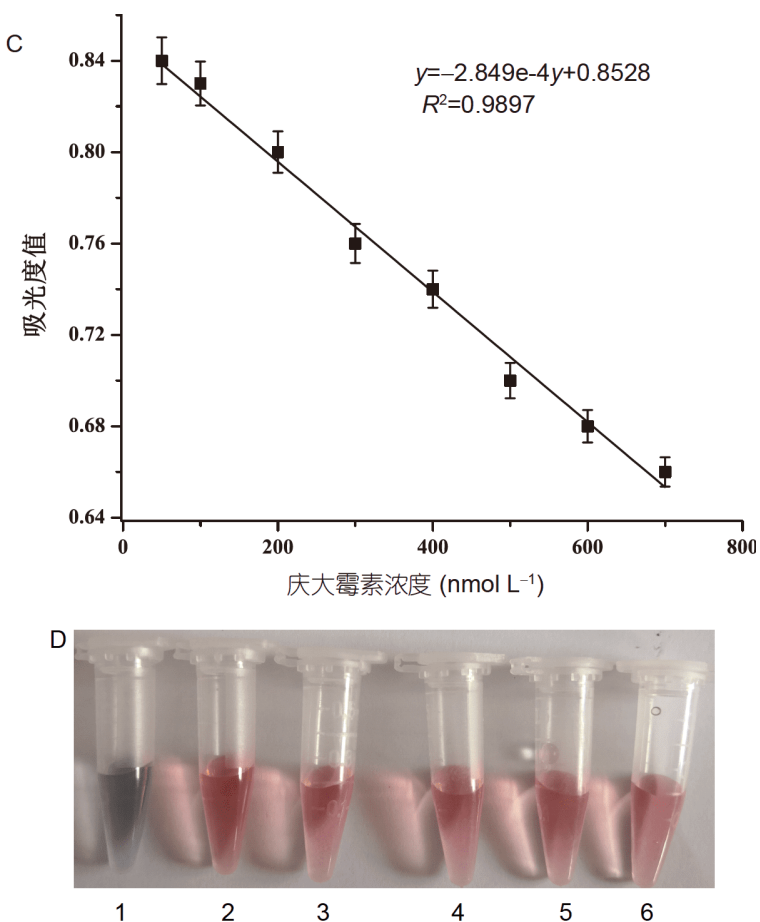

图 5 AuNPs比色法检测庆大霉素. A: 在不同浓度庆大霉素存在下, AuNPs的聚集和颜色变化情况 $(1: 0 \mathrm{nmol} / \mathrm{L} ; 2: 50 \mathrm{nmol} / \mathrm{L}$; 3: $100 \mathrm{nmol} / \mathrm{L} ; 4: 200 \mathrm{nmol} / \mathrm{L} ; 5: 300 \mathrm{nmol} / \mathrm{L} ; 6: 400 \mathrm{nmol} / \mathrm{L} ; 7: 500 \mathrm{nmol} / \mathrm{L} ; 8: 600 \mathrm{nmol} / \mathrm{L} ; 9: 700 \mathrm{nmol} / \mathrm{L}) ; \mathrm{B}:$ 在不同浓度庆大霉 素存在下, AuNPs溶液在 NaCl溶液中的UV-Vis光谱图; C: AuNPs溶液在 $520 \mathrm{~nm}$ 处的吸光值与庆大霉素浓度的线性关系图; D:

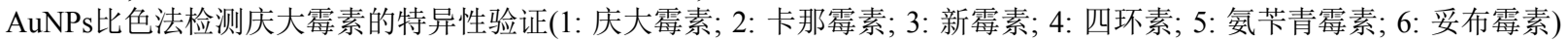

Figure 5 AuNPs-based colorimetric assay of gentamicin. A: The aggregation of AuNPs and corresponding color change in the presence of different concentrations of gentamicin $(1: 0 \mathrm{nmol} / \mathrm{L} ; 2: 50 \mathrm{nmol} / \mathrm{L} ; 3: 100 \mathrm{nmol} / \mathrm{L} ; 4: 200 \mathrm{nmol} / \mathrm{L} ; 5: 300 \mathrm{nmol} / \mathrm{L} ; 6: 400 \mathrm{nmol} / \mathrm{L} ; 7: 500 \mathrm{nmol} / \mathrm{L} ; 8: 600 \mathrm{nmol} / \mathrm{L}$; 9: $700 \mathrm{nmol} / \mathrm{L}$ ). B: The UV-vis spectra of AuNPs in NaCl solution in the presence of different concentrations of gentamicin. C: The linear relationship between the absorbance of AuNPs at $520 \mathrm{~nm}$ and the concentration of gentamicin. D: Specificity verification of AuNPs colorimetric method for detection of tobramycin (1: gentamicin; 2: kanamycin; 3: neomycin; 4: tetracycline; 5: ampicillin; 6: tobramycin)

表 2 AuNPs比色法检测牛奶样品中庆大霉素的结果及回 收率

Table 2 The results and recovery of gentamicin detection in milk samples by using AuNPs-based colorimetric assay

\begin{tabular}{ccc}
\hline \multirow{2}{*}{$\begin{array}{c}\text { 加入庆大需素浓度 } \\
(\mathrm{nmol} / \mathrm{L})\end{array}$} & \multicolumn{2}{c}{ 牛奶样品 $(n=3)$} \\
\cline { 2 - 3 } & 检测浓度 $(\mathrm{nmol} / \mathrm{L})$ & 回收率 $(\%)$ \\
\hline 50 & $43.27 \pm 3.37$ & 86.54 \\
100 & $92.38 \pm 8.26$ & 92.38 \\
200 & $187.1 \pm 13.41$ & 93.56 \\
500 & $455.3 \pm 16.14$ & 91.06 \\
\hline
\end{tabular}

霉素的离心管中AuNPs溶液变为蓝灰色，而加入其他 抗生素的离心管中AuNPs溶液颜色未发生明显变化. 由此可见, 该方法检测庆大霉素具有良好的特异性.

为了进一步验证AuNPs比色法在检测实际样品中 庆大霉素时的实用性，将该方法应用于对人工污染庆
大霉素的牛奶的检测. 如表2所示, 当牛奶中添加的庆 大霉素浓度为 $50,100,200$ 和 $500 \mathrm{nmol} / \mathrm{L}$ 时，检测结果 与庆大霉素标准品的实验结果基本一致, 说明牛奶样 品基质成分对检测结果影响较小，即该方法可以用于 实际牛奶样品中庆大霉素的检测.

\section{3 讨论}

庆大霉素是应用较为广泛的一种氨基糖苗类抗生 素, 除应用于临床治疗外, 还常被用作兽用饲料的添加 物. 但是, 随着庆大霉素毒副作用的阐明, 在临床方面 已逐步被其他抗生素所取代，而在其他领域则仍有使 用，而不规范使用最终会将庆大霉素残留带到食品和 环境中, 对人体健康产生严重危害. 因此, 建立高灵敏 度、高特异性、简便的庆大霉素检测方法十分必要. 
目前以仪器分析为核心手段的方法虽然具有稳定性 好、特异性高等优点，但无法解决市场监管等场合现 场快速检测的需求. 以适配体为核心元件的生物传感 器为快速简便、高灵敏度、高特异性检测庆大霉素提 供了可能.

SELEX技术是应用库容量达到 $10^{12} \sim 10^{15}$ 的随机寡 核苷酸文库, 结合PCR扩增技术, 以指数的方式富集与 靶分子高特异性结合的寡核苷酸, 最终经过多轮体外 筛选获得高特异性、高亲和力的适配体. 目前，针对 小分子靶标的笁选方法主要有磁珠SELEX、氧化石 墨烯SELEX (GO-SELEX)及亲和层析SELEX等. 相比 其他几种SELEX技术，亲和层析SELEX在操作上虽略 复杂，但在高效分离与靶标结合的 $\mathrm{ssDNA}$ 和游离 ssDNA方面具有明显的优势, 为篮选出高亲和力的适 配体提供了更大的可能. 本文以庆大霉素为靶标，利 用亲和层析SELEX方法，通过九轮篮选，获得了 34 条 与庆大霉素有亲和力的适配体. 利用DNAMAN和 Mfold软件分别对其同源性和二级结构进行分析，将 其分为六大家族，并选择序列较多家族中结构最稳定 的序列作为庆大霉素适配体的候选序列. 这种方式可 以大大降低实验工作量，有利于快速篮选出最优适配 体序列，但也有可能遗漏部分具有优良特性的适配体 序列. 通过苂光分析法测定了 $5,8,22,26$ 号适配体序 列的解离常数，其中 26 号适配体 $K_{\mathrm{d}}$ 值达到 $14.00 \pm$ $3.34 \mathrm{nmol} / \mathrm{L}$, 亲和力最大. 本文采用亲和层析-SELEX 笁选获得的适配体亲和力高于之前用磁珠-SELEX和 GO-SELEX所得到的适配体, 验证了亲和层析SELEX
的优势. 同时, 特异性实验表明, 26 号适配体不仅对庆 大霉素具有高亲和力, 还具有很高的特异性. 因此将 26 号适配选作最佳的庆大霉素适配体, 命名为Ap-26. 以 Ap-26为识别元件构建的AuNPs比色法为庆大霉素提 供了一种快速、简便和低成本的检测方法. 当庆大霉 素的浓度在50 700 nmol/L范围内时, 在 $520 \mathrm{~nm}$ 处的吸 光值与庆大霉素浓度之间存在线性关系, 检测限为 $11.50 \mathrm{nmol} / \mathrm{L}$. 将该方法应用于牛奶样品中庆大霉素的 检测，结果表明该方法能够成功实现实际样品的检测. 与已报道的其他方法相比，该方法具有简便、快捷、 灵敏度高等优势(表3). 基于Ap-26的AuNPs比色法是 一种可以在 $1 \mathrm{~h}$ 内完成的凭肉眼观测即可进行半定量 分析庆大霉素的方法, 为食品安全市场监管和环境监 测等提供了有效的手段.

通常由SELEX篮选获得的适配体长度为70 130 个核苷酸，其两端为用于PCR扩增时与引物结合的固 定序列，中间为随机序列. 固定序列和随机序列均有 可能在形成核酸空间结构时参与靶标的结合位点. 该 长度的ssDNA合成成本还相对比较高，同时单链核酸 的空间构象具有较大的可变性，在不同 $\mathrm{pH}$ 和离子条件 下有可能发生改变，从而影响到适配体与靶标的结合 力. 由于与靶标结合及稳定结合位点的核苷酸只是适 配体序列中的一小部分，对适配体进行截短，不仅可 以减少在应用过程中的成本，还能提高靶标与适配体 之间的结合效率，降低溶液条件对结合的干扰. 预期 在Ap-26的基础上利用分子对接预测的结合位点结果 可为Ap-26的截短和优化提供理论基础.

表 3 本文建立的庆大霉素检测方法与其他已报道方法的比较

Table 3 Comparison of the proposed method with other reported methods for gentamicin detection

\begin{tabular}{|c|c|c|c|c|}
\hline 方法 & 原理 & 样品 & 检测限 & 参考文献 \\
\hline 微生物检测法 & $\begin{array}{c}\text { 根据庆大霉素对短小芽孢杆菌生命活动的抑制来定量 } \\
\text { 检测庆大霉素的残留 }\end{array}$ & 兔血清 & $0.02 \mu \mathrm{g} / \mathrm{mL}$ & {$[8]$} \\
\hline 免疫检测法 & $\begin{array}{c}\text { 利用庆大霉素抗体为识别元件, 分别通过苂光偏振分析 } \\
\text { 法、酶联免疫吸附法来测定庆大霉素 }\end{array}$ & 动物源性样品 & $\begin{array}{r}0.17 \mu \mathrm{g} / \mathrm{mL} 、 \\
0.001 \mu \mathrm{g} / \mathrm{mL}\end{array}$ & [9] \\
\hline 理化分析法 & $\begin{array}{c}\text { 利用高效液相色谱蒸发光散射检测器对庆大霉素进行 } \\
\text { 检测 }\end{array}$ & 鱼腥草 & $150 \mu \mathrm{g} / \mathrm{mL}$ & {$[10]$} \\
\hline 比色法 & $\begin{array}{c}\text { 建立基于半胱胺修饰的金纳米粒子比色法检测庆大 } \\
\text { 霉素 }\end{array}$ & 水 & $7.17 \times 10 \mathrm{ng} / \mathrm{mL}$ & {$[1]$} \\
\hline 比色法 & $\begin{array}{c}\text { 利用适配体对金纳米粒子的保护和庆大霉素的竞争置 } \\
\text { 换作用 }\end{array}$ & 牛奶 & $\begin{array}{c}11.50 \mathrm{nmol} / \mathrm{L} \\
(6.62 \mathrm{ng} / \mathrm{mL})\end{array}$ & 本文 \\
\hline
\end{tabular}




\section{参考文献}

1 Gukowsky J C, Tan C, Han Z, et al. Cysteamine-modified gold nanoparticles as a colorimetric sensor for the rapid detection of gentamicin. J Food Sci, 2018, 83: 1631-1638

2 Liu Q, Mu H, Sun C, et al. Highly specific determination of gentamicin by induced collapse of Au-lipid capsules. RSC Adv, 2016, 6: 1448314489

3 Jian X Y, Deng Z X, Sun Y H. Aminoglycoside gentamicin research: fundamentalprogressand new application prospects (in Chinese). Chin J Biotech, 2015, 31: 829-844 [简心韵, 邓子新, 孙宇辉. 氨基糖苷抗生素庆大霉素: 基础研究的新进展及应用研究的新潜力. 生物工程学报, 2015, 31: 829-844]

$4 \mathrm{Hu} \mathrm{J} \mathrm{G}, \mathrm{Fu} \mathrm{Y,} \mathrm{Xu} \mathrm{J} \mathrm{J,} \mathrm{et} \mathrm{al.} \mathrm{Altered} \mathrm{gene} \mathrm{expression} \mathrm{profile} \mathrm{in} \mathrm{a} \mathrm{rat} \mathrm{model} \mathrm{of} \mathrm{gentamicin-induced} \mathrm{ototoxicity} \mathrm{and} \mathrm{nephrotoxicity,} \mathrm{and} \mathrm{the} \mathrm{potential}$ role of upregulated Ifi44 expression. Mol Med Rep, 2017, 16: 4650-4658

5 Zhao J, Liu T, Cai B Y, et al. Determination of gentamicin in raw milk by high-performance liquid chromatopraphy (in Chinese). Food Res Dev, 2015, 36: 94-97 [赵静, 刘婷, 蔡柏岩, 等. HPLC法测定原料乳中庆大霉素残留的研究. 食品研究与开发, 2015, 36: 94-97]

6 Wang L Z, Li L L, Zhao Y, et al. Development of gentamicin semi quantitative colloidal gold test strip (in Chinese). China Feed, 2013, 20: 16-18 [王丽哲, 王丽丽, 赵瑜, 等. 牛奶中庆大霉素半定量胶体金试纸条的研制. 中国饲料, 2013, 20: 16-18]

7 Ruan Z Y, Chen X, Sun W, et al. Isolation and identification of cow mastitis pathogenic bacteria and disk diffusion test (in Chinese). Anim Husb Veter Med, 2018, 50: 105-109 [阮智杨, 陈新, 孙雯, 等. 奶牛乳房炎病原菌的分离鉴定及药物敏感试验. 畜牧与兽医, 2018, 50: 105-109]

8 Yin Q S, Ren C L, Chen D L, et al. Determination of low concentration gentamycin in rabbit's serum by microbiological method (in Chinese). Chin J Pharm Anal, 2006, 26: 1811-1813 [尹情胜, 任翠丽, 陈殿良, 等. 微生物法测定免血清中低浓度的庆大需素. 药物分析杂志, 2006, 26: 1811-1813]

9 Li C, Zhang Y, Eremin S A, et al. Detection of kanamycin and gentamicin residues in animal-derived food using IgY antibody based ic-ELISA and FPIA. Food Chem, 2017, 227: 48-54

10 Wang J W, Gong X H, Fan Q, et al. Determination of gentamicin illegally added in houttuynia injection by HPLC-ELSD and UPLC-MS /MS (in Chinese). Chin J Veter Drug, 2018, 52: 45-51 [王静文, 龚旭昊, 范强, 等. HPLC-ELSD法联合UPLC-MS/MS法检测鱼腥草注射液中非法添加 的庆大霉素. 中国兽药杂志, 2018, 52: 45-51]

11 Tuerk C, Gold L. Systematic evolution of ligands by exponential enrichment: RNA ligands to bacteriophage T4 DNA polymerase. Science, 1990, 249: $505-510$

12 Ellington A D, Szostak J W. In vitro selection of RNA molecules that bind specific ligands. Nature, 1990, 346: 818-822

13 Wang Y, Li S Z, You Y, et al. Application progress of aptamer in biotoxin detection (in Chinese). J Henan Agricult Sci, 2018, 47: 1-6 [王耀, 李少 珍, 游一, 等. 适配体在生物毒素检测中的应用进展. 河南农业科学, 2018, 47: 1-6]

14 Bauer M, Macdonald J, Henri J, et al. The application of aptamers for immunohistochemistry. Nucl Acid Therap, 2016, 26: 120-126

15 Han K, Liang Z, Zhou N. Design strategies for aptamer-based biosensors. Sensors, 2010, 10: 4541-4557

16 Jauset-Rubio M, El-Shahawi M S, Bashammakh A S, et al. Advances in aptamers-based lateral flow assays. TrAC Trends Anal Chem, 2017, 97: 385-398

17 Sun H, Tan W, Zu Y. Aptamers: versatile molecular recognition probes for cancer detection. Analyst, 2015, 141: 403-415

18 Zhou N, Wang J, Zhang J, et al. Selection and identification of streptomycin-specific single-stranded DNA aptamers and the application in the detection of streptomycin in honey. Talanta, 2013, 108: 109-116

19 Song K M, Cho M, Jo H, et al. Gold nanoparticle-based colorimetric detection of kanamycin using a DNA aptamer. Anal Biochem, 2011, 415: $175-181$

20 Han X, Zhang Y, Nie J, et al. Gold nanoparticle based photometric determination of tobramycin by using new specific DNA aptamers. Microchim Acta, 2018, 185: 4

21 Dolati S, Ramezani M, Nabavinia M S, et al. Selection of specific aptamer against enrofloxacin and fabrication of graphene oxide based label-free fluorescent assay. Anal Biochem, 2018, 549: 124-129

22 Mehta J, Rouah-Martin E, Van Dorst B, et al. Selection and characterization of PCB-binding DNA aptamers. Anal Chem, 2012, 84: 1669-1676 
23 Zhang Y, You Y, Xia Z, et al. Graphene oxide-based selection and identification of ofloxacin-specific single-stranded DNA aptamers. RSC Adv, 2016, 6: 99540-99545

$24 \mathrm{Xu} \mathrm{Y,} \mathrm{Sun} \mathrm{L,} \mathrm{Huang} \mathrm{X,} \mathrm{et} \mathrm{al.} \mathrm{A} \mathrm{label-free} \mathrm{and} \mathrm{signal-on} \mathrm{electrochemical} \mathrm{aptasensor} \mathrm{for} \mathrm{ultrasensitive} \mathrm{kanamycin} \mathrm{detection} \mathrm{based} \mathrm{on} \mathrm{exonuclease}$ recycling cleavage. Anal Methods, 2016, 8: 726-730

25 Zheng D, Zhu X, Zhu X, et al. An electrochemical biosensor for the direct detection of oxytetracycline in mouse blood serum and urine. Analyst, 2013, 138: 1886-1990

$26 \mathrm{Xu} \mathrm{Y,} \mathrm{Han} \mathrm{T,} \mathrm{Li} \mathrm{X,} \mathrm{et} \mathrm{al.} \mathrm{Colorimetric} \mathrm{detection} \mathrm{of} \mathrm{kanamycin} \mathrm{based} \mathrm{on} \mathrm{analyte-protected} \mathrm{silver} \mathrm{nanoparticles} \mathrm{and} \mathrm{aptamer-selective} \mathrm{sensing}$ mechanism. Anal Chim Acta, 2015, 891: 298-303

27 Ramezani M, Danesh N M, Lavaee P, et al. A selective and sensitive fluorescent aptasensor for detection of kanamycin based on catalytic recycling activity of exonuclease III and gold nanoparticles. Sens Actuat B-Chem, 2016, 222: 1-7

28 Leung K H, He H Z, Chan D S H, et al. An oligonucleotide-based switch-on luminescent probe for the detection of kanamycin in aqueous solution. Sens Actuat B-Chem, 2013, 177: 487-492

29 Nasir A, Kausar A. A review on materials derived from polystyrene and different types of nanoparticles. Polymer-Plastics Tech Eng, 2015, 54: 1819-1849

30 Chen M J, Yang W T, Yin M Z. Synthesis and applications of nanoparticles in biology (in Chinese). Prog Chem, 2012, 24: 2403-2414 [陈梦君, 杨万泰, 尹梅贞. 纳米粒子的分类合成及其在生物领域的应用. 化学进展, 2012, 24: 2403-2414]

31 Verma A, Stellacci F. Effect of surface properties on nanoparticle-cell interactions. Small, 2010, 6: 12-21

32 Atmane Y A, Sicard L, Lamouri A, et al. Functionalization of aluminum nanoparticles using a combination of aryl diazonium salt chemistry and iniferter method. J Phys Chem C, 2017, 117: 26000-26006

33 Häkkinen H. The gold-sulfur interface at the nanoscale. Nat Chem, 2012, 4: 443-455

34 Mirahadi M, Ghanbarzadeh S, Ghorbani M, et al. A review on the role of lipid-based nanoparticles in medical diagnosis and imaging. Therap Deliver, 2018, 9: 557-569

35 Bagheri H, Shirzadmehr A, Rezaei M, et al. Determination of tramadol in pharmaceutical products and biological samples using a new nanocomposite carbon paste sensor based on decorated nanographene/tramadol-imprinted polymer nanoparticles/ionic liquid. Ionics, 2018, 24: $833-843$

36 Jørgensen M, Grönbeck H. The site-assembly determines catalytic activity of nanoparticles. Angew Chem Int Ed, 2018, 57: 5086-5089

37 Hu X, Chang K, Wang S, et al. Aptamer-functionalized AuNPs for the high-sensitivity colorimetric detection of melamine in milk samples. PLoS ONE, 2018, 13: e0201626 


\title{
Selection, characterization and application of gentamicin-specific single-stranded DNA Aptamers
}

\author{
WU MengMeng ${ }^{1}$, HAN XuYan ${ }^{1}$, CAI RongFeng ${ }^{1}$, LI MaoLin ${ }^{2}$, TIAN YaPing $^{1} \&$ ZHOU NanDi ${ }^{1}$ \\ 1 Key Laboratory of Carbohydrate Chemistry and Biotechnology, Ministry of Education, School of Biotechnology, Jiangnan University, \\ Wuxi 214122, China; \\ 2 Department of Clinical Laboratory, Yixing Cancer Hospital, Yixing 214200, China
}

\begin{abstract}
Gentamicin is a widely used aminoglycoside antibiotic which acts effectively against a wide spectrum of bacteria, especially Gram-negative bacteria. Due to its serious side effect, the abuse of gentamicin will be harmful to the environment and human health. Therefore, it is important to estbalish a highly sensitive assay of gentamicin for the supervision of food market and environment monitoring. Herein, Sepharose affinity chromatography-based systematic evolution of ligands by exponential enrichment (SELEX) was carried out to screen gentamicin-specific aptamers. Through nine rounds of selection and subsequent cloning and sequencing, 34 aptamer sequences were screened from a 79-nucleotides initial ssDNA library. These sequences were classified into 6 families according to homology analysis. Then Mfold software was employed to predict the secondary structures and the stability of these aptamers. Aptamers with a high stability were chosen to determine the dissociate constants $\left(K_{\mathrm{d}}\right)$. An aptamer named Ap-26 was finally screened, whose $K_{\mathrm{d}}$ was determined to be only $14.00 \pm 3.34 \mathrm{nmol} / \mathrm{L}$, indicating very high affinity for gentamicin. Molecular docking was carried out by Autodock 4.0 software. The result shows that nucleotides No. 17-20 and 54-56 of the aptamer were the binding sites towards gentamicin. Finally, Ap-26 was used as recognition element to construct gold nanoparticles (AuNPs)-based colorimetric assay for gentamicin, which further verified the practicability of the aptamer. Within the range of $50-700 \mathrm{nmol} / \mathrm{L}$, a linear relationship was derived between the absorbance of AuNPs solution at $520 \mathrm{~nm}$ and the concentration of gentamicin. The detection limit was estimated to be $11.50 \mathrm{nmol} / \mathrm{L}$. The assay was then applied to detect gentamicin in milk samples. The results show that the matrix of milk hardly affects the detection, and the recovery of gentamicin is higher than $90 \%$. Therefore, the screened aptamer and the established assay may have great prospect in the detection of gentamicin.
\end{abstract}

gentamicin, SELEX, aptamer, affinity chromatography, gold nanoparticles

doi: $10.1360 / \mathrm{N} 052018-00238$ 